\title{
Desarrollo sostenible: Impacto ambiental y retos de los hospitales ante la Covid-19
}

\section{Sustainable development: Environmental impact and challenges for hospitals in the face of Covid-19}

$\mathrm{DOI}$

\author{
Javier Isidro Rodríguez-López ${ }^{1, a}$, Rosa Diaz-Manchay ${ }^{2, b}$, José Manuel Herrera-Paredes ${ }^{1, b}$
}

https://doi.org/10.35434/rcmhnaaa.2021.14Sup1.1190

\section{Señor editor:}

Los gobiernos a nivel mundial en los últimos 50 años han generado conjuntamente diversas agendas, compromisos y planes que abordan las necesidades derivadas de las interacciones sociales, económicas y ambientales; tal es el caso de Alma Ata, Agenda 21, y los Objetivos de Desarrollo Sostenible (ODS); entre otras, lo que conlleva a establecer responsabilidades políticas en salud relacionadas con el desarrollo sostenible en cada continente, región y país. Sin embargo, la actual crisis mundial generada por el Coronavirus (COVID-19) ha dificultado la continuidad de estos compromisos, priorizando el combate a este terrible mal y que hasta la fecha no existe una aparente solución.

Sin considerar la pandemia que aqueja al mundo por COVID-19, surgía la necesidad de una gestión adecuada de los residuos sólidos y el manejo de sustancias químicas, así como la capacitación continua del personal de salud sobre prácticas ambientales saludables, que contribuyan al ahorro de luz eléctrica y agua en los hospitales ${ }^{(1,2)}$. Pero, el origen del COVID-19 y sus riesgos potenciales para la salud aumentó el uso de la mascarilla facial; así como otro tipo de materiales en uniformes, guantes, caretas, entre otros; lo que trae el problemático incremento de desechos médicos, manifestándose un déficit de competencias su manejo adecuado, que sin duda originan causas fundamentales de enfermedades graves y problemas ambientales ${ }^{(3)}$. Además, a medida que continúa la pandemia se ha analizado el consumo de recursos necesarios para la producción de equipos de protección personal y kits de prueba ${ }^{(4)}$.

Ante ello, el Programa de Naciones Unidas para el Medio Ambiente (PNUMA) hace recordar que la gestión inadecuada de los residuos médicos y peligrosos derivados de esta crisis sanitaria podría desencadenar un efecto rebote y otras consecuencias para la salud, por lo que su manejo y eliminación segura resulta vital ${ }^{(5)}$. De acuerdo a lo evidenciado en un estudio en Brasil, la cantidad de residuos clínicos generados aumentaron para mayo de $2020^{(6)}$. A partir de ello, establecieron que los residuos clínicos deben estar debidamente regulados para evitar cualquier infección no deseada y consecuencias adversas para la salud y el ambiente, ya que los deshechos clínicos posiblemente contengan tejidos humanos, sangre, fluidos corporales, drogas de excreción, agujas y otros materiales contaminados ${ }^{(7)}$.

En ese sentido, en Latinoamérica aún no se realizan estudios sobre el impacto ambiental de los hospitales y los retos sobre la sostenibilidad ante la COVID-19. Se tiene en cuenta la responsabilidad de las instituciones de salud y el uso de elementos de protección personal e insumos necesarios para desarrollo de las acciones de atención de la salud; sin embargo, no se razona sobre el impacto ambiental importante que se esto produce; dado que, la generación de contaminantes aumenta el riesgo de contraer comorbilidad para la población vulnerable que se encuentra en los servicios.

A pesar de la existencia de parámetros, normativas y protocolos de la gestión integral del manejo de residuos sólidos y químicos desde su uso, segregación, disposición,

\author{
FILIACIÓN \\ 1. Universidad de Guanajuato Celaya, \\ Guanajuato, México. \\ 2. Universidad Católica Santo Toribio de \\ Mogrovejo, Chiclayo, Perú. \\ a. Estudiante de Doctorado en Ciencias de la \\ b. Doctor en Ciencias de Enfermería. \\ ORCID \\ 1. Rodríguez-López Javier Isidro \\ 0000-0002-1907-4152 \\ Diaz-Manchay Rosa
$0000-0002-2333-7963$ \\ 3. Heorrera-Paredes José Manuel \\ 0000-0001-7631-5904 \\ CORRESPONDENCIA \\ Javier Isidro Rodríguez López. \\ Dirección: Tabachines 501 Jardines de Celaya \\ 2da sección, Celaya Guanajuato, México. \\ Teléfono: +573112667903 \\ EMAIL \\ ji.rodriguezlopez@ugto.mx \\ CONFLICTOS DE INTERÉS \\ Los autores niegan conflictos de interés. \\ FINANCIAMIENTO \\ Autofinanciamiento. \\ REVISIÓN DE PARES \\ Recibido: 10/06/2021 \\ Aceptado: 15/08/2021

\section{COMO CITAR} \\ Rodríguez-López, J., Diaz-Manchay, R., \& \\ Herrera-Paredes, J. Desarrollo sostenible: \\ Impacto ambiental y retos de los hospitales \\ ante la Covid-19. Revista Del Cuerpo Médico \\ Hospital Nacional Almanzor Aguinaga Asenjo, \\ 2021,14 ( S u p 1), $100-102$. \\ https://doi.org/10.35434/rcmhnaaa.2021.14S \\ up1.1190
}

Esta obra está bajo una Licencia Creative Commons Atribución 4.0 Internacional. Versión Impresa: ISSN: 2225-5109 Versión Electrónica: ISSN: 2227-4731 Cross Ref. DOI: 10.35434/rcmhnaaa OJS: https://cmhnaaa.org.pe/ojs 
recolección y disposición final. Así en Perú, se tiene un manual de recomendaciones para el manejo de residuos sólidos durante la epidemia de COVID-19 ${ }^{(8)}$, en este contexto es que nos con lleva a reflexionar ¿cómo se maneja la cantidad de residuos generados por COVID-19 en su almacenamiento, manejo sanitario, y transporte para minimizar los riesgos para usuarios y colaboradores?, es decir de que manera lo ponen en práctica. Sin dejar de lado el incremento en costos para los hospitales por las compras de insumos necesarios para la atención, dejando de lado que estos cumplan con los criterios de consumo responsable por las pocas alternativas de proveedores.

Asimismo, la infraestructura también se ve afectada debido al mejoramiento de la capacidad hospitalaria denominándose "Reconversión de los servicios de salud"; es decir, se trata de ampliar las zonas exclusivas para pacientes con COVID-19 $9^{(9)}$, eliminando espacios e implementando otros para camas de Unidades de Cuidados Intensivos y nuevos respiradores; y es aquí nuevamente dónde se presenta la oportunidad de analizar la reconversión; ya que los cambios en la iluminación y ventilación afectan el confort para el paciente y trabajadores. Al igual, el incremento de las sustancias químicas de uso para desinfección permanente requiere de una gestión integral desde la compra hasta su disposición final. Sangkham ${ }^{(3)}$, manifiesta que la eliminación de los desechos médicos ante la pandemia en Asia se debe considerar cuidadosamente la estandarización, los procedimientos, las pautas y la implementación estricta de la gestión de desechos médicos ocasionados por la atención de la pandemia, con el fin de reducir el riesgo que el agente patógeno del COVID-19 se propague al medio ambiente dentro de los hospitales y áreas públicas.

Por otro lado, evidentemente se ha incrementado el consumo energético, hídrico y productos farmacéuticos pues estos recursos nunca paran de manejarse en las instituciones de salud, el primero; se puede haber incrementado por aumento de respiradores, camas y la reconversión de servicios de salud; el consumo de agua, puede haberse incrementado, además dejando al lado un tópico poco analizado como los vertimientos y su control debido al aumento de sustancias químicas y no saber la biodisponibilidad del virus que sería aspectos a profundizar, para el manejo de medicamentos en zonas de cuidado critico se ha aumentado ampliamente, incluyendo el oxígeno, generando falta de estos insumos en diversos países.

Otros de los componentes que tienen presencia en este fenómeno son los aspectos relacionados con la preparación y manejo de alimentos para los pacientes; así como la disposición de los residuos orgánicos, mismos que tuvieron contacto con pacientes en las zonas de COVID-19, asimismo la disposición de espacios seguros para la alimentación de los trabajadores y visitantes; sin olvidar que la movilidad de pacientes que ingresan o se trasladan de hospitales requieren adecuación de los vehículos, así como la seguridad personal de los conductores.

Por último, aspecto medular para el desarrollo de la sostenibilidad de los hospitales, es el liderazgo ambiental, tópico débil desde los procesos de formación profesional por lo cual hace más difícil el manejo para lograr esa cultura sostenible ${ }^{(2,10)}$, ahora en tiempo de pandemia el reto es mayor ya que el enfoque está centralizado en el cuidado directo, logrando que no se cuente con los espacios y tiempos para generar competencias en sostenibilidad. Además, el cumplimiento de los Objetivos de Desarrollo Sostenible (ODS) se ve afectado, colocando en riesgo su compromiso, inversión y logro de metas relacionadas con el manejo hídrico, el consumo energético, el consumo sostenible y comunidades sostenibles ${ }^{(11)}$, así disminuyendo los beneficios para las sociedades.

Todo lo mencionado debe comprenderse desde la perspectiva de experiencias, realidades y capacidades de cada una de las instituciones de salud; ya sean de primer, segundo y tercer nivel de atención, sin importar la ubicación geográfica de los hospitales, la exigencia o laxitud de la normatividad local, además de los compromisos dados en lo ambiental para su funcionamiento y/o gestión ante procesos de acreditación. Por ello se invita a no generalizar, pero si a considerar e investigar sobre los aspectos mencionados para la gestión e implementen de políticas y estrategias de sostenibilidad ambiental con el fin de salvaguardar la salud de las vidas del planeta donde estamos los humanos inmersos.

Finalmente, Capoor y Parida ${ }^{(12)}$ establecen que el paso clave en la gestión de residuos de COVID-19 es su adecuada segregación. Así, al igual que para la gestión de COVID-19 se propone "Identificar Aislar y Tratar", lo mismo ocurre con los residuos de COVID-19: identificar el generador, aislar, es decir, gestionar por separado en los contenedores de residuos designados y finalmente tratarlos de forma adecuada, siguiendo estrictamente la regulación declarada para su control. Por otra parte, unas de las soluciones alternativas probables para el manejo de estos residuos es el uso de tecnologías simples con el uso de rayos UV, el control de la humedad y la temperatura en los sitios de almacenamiento de este tipo de residuos ${ }^{(13)}$.

\section{REFERENCIAS BIBLIOGRÁFICAS}

1. Rojas H, Díaz M, Muro I, Díaz R. Sostenibilidad ambiental de la práctica clínica, una nueva visión para enfermería. ACC CIETNA: Revista de la Escuela de Enfermería [Internet]. 2020 [citado 30 ene

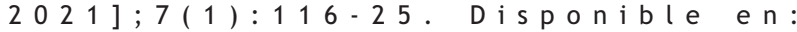
http://revistas.usat.edu.pe/index.php/cietna/article/view/353/7 83.

2. Restrepo HF, Rodríguez JI, Aponte SM. Valoración de la comunicación ambiental en las instituciones de salud de la cámara sectorial de salud de la ANDI - Colombia. Repert. Med. Cir. [Internet]. 2020 [citado 18 ago 2021];30(2):125-33. Disponible en: https://revistas.fucsalud.edu.co/index.php/repertorio/article/vie w/1018.

3. Sangkham S. Face mask and medical waste disposal during the novel COVID-19 pandemic in Asia. Case Studies in Chemical and Environmental Engineering [Internet]. 2020 [cited 2021 jan 30 ] ; 2: 100052 . Ava il a ble from. https://doi.org/10.1016/j.cscee.2020.100052.

4. Klemeš JJ, Fan YV, Jiang P. The energy and environmental footprints of COVID-19 fighting measures - PPE, disinfection, supply chains. Energy (Oxf). 2020;211:118701. doi: 10.1016/j.energy.2020.118701.

5. Organización de las Naciones Unidad. ¿Cómo ha de ser la gestión de residuos durante la pandemia del coronavirus? [Internet]. Noticias ONU; 2020 [citado 5 feb 2021]. Disponible en: https://news.un.org/es/story/2020/04/1472202.

6. Urban, RC y Nakada, LYK. COVID-19 pandemic: Solid waste and environmental impacts in Brazil. Sci Total Environ. 2021;755(Pt 1):142471. doi: $10.1016 /$ j.scitotenv.2020.142471.

7. Agamuthu P, Barasarathi J. Clinical waste management under COVID- 
19 scenario in Malaysia. Waste Manag Res. 2020;39(1_suppl):18-26. doi:10.1177/0734242X20959701.

8. Ministerio del ambiente de Perú. Guía / Manual: Recomendaciones para el manejo de residuos sólidos durante la epidemia de COVID-19 [Internet]. Lima: Sistema Nacional de Información Ambiental - SINIA 2020 [citado 30 ene 2021];1-17. Disponible en: https://sinia.minam.gob.pe/documentos/recomendaciones manejo-residuos-solidos-durante-epidemia-covid-19.

9. Castañeda-Hernández DM, Rodríguez López JI. COVID 19 - La pandemia. Una mirada a sus impactos en Latinoamérica. Revista Venezolana de Gerencia. 2020;25(91):746-753. doi: https://doi.org/10.37960/rvg.v25i91.33162.

10. Rodríguez JI, Gonzalez M, Aponte SM. Transversalización de la Salud ambiental hospitalaria en el currículo de programas de Ciencias de la Salud a través de las TICS en el año 2018. En: Antolí Martínez J, Lledó Carreres A, Pellín Buades N, et al. Redes de Investigación e Innovación en Docencia Universitaria [Internet]. Alicante: Universidad de
Alicante; 2019 [citado 30 ene 2021]. p.577-89. Disponible en: https: / /rua.ua.es/dspace/bitstream/10045/99177/1/RedesInvestigacion-Innovacion-Docencia-Universitaria-2019-51.pdf.

11. Rodríguez J, Aponte S, González M, Dimate A, Muñoz E. Avances de la vinculación del componente ambiental y hospitales verdes en carreras de ciencias de la salud. En: Restrepo Guerrero H, Rodríguez López J, et al. Hospitales verdes: Un nuevo desafío para América Latina [Internet]. Bogotá: Fundación Universitaria del Área Andina; 2020 [citado 30 ene 2021]. p.251-86. Disponible en: https://digitk.areandina.edu.co/handle/areandina/3825.

12. Capoor MR, Parida A. Current perspectives of biomedical waste management in context of COVID-19". Indian J Med Microbiol. 2021;39(2),171-8. doi: 10.1016/j.ijmmb.2021.03.003.

13. Bellizzi S, Kamal SA, Newir AE, Pichierri G, Salaris P, Pinto S, et al. Simple technology for COVID-19 medical solid waste treatment in low-resourced settings. J Glob Health. 2020;10(2):020373. doi: 10.7189/jogh.10.020373 